\title{
REVISIT OF THE WALL EFFECT ON THE SETTLING OF CYLINDRICAL PARTICLES IN THE INERTIAL REGIME
}

\author{
Raymond Lau ${ }^{1}$, Meer Saiful Hassan, Wenyin Wong, and Tao Chen \\ School of Chemical and Biomedical Engineering (SCBE) \\ Nanyang Technological University (NTU) \\ Singapore
}

\begin{abstract}
The wall effect on the settling of cylindrical particles in the inertial regime is revisited. This study covers particle Reynolds number from 600-16100 and particle length-to-diameter ratio $(l / d)$ from 4-21. The wall factor $\left(U_{t} / U_{t, \infty}\right)$ is found to decrease initially as the particle-tocolumn diameter ratio $(d / D)$ increases. When wall effect becomes significant, $U_{t} / U_{t, \infty}$ starts to increase with an increase in $d / D$ due to a change of the particle orientation during settling. After $U_{t} / U_{t, \infty}$ reaches a maximum, it decreases to 0 when $d / D$ increases to 1 . A semi-empirical model is developed to predict the wall effect of settling of cylindrical particles over the whole range of $d / D$. The model shows good prediction to the experimental results and matches the wall factor models developed for spherical particles when $l / d$ approaches 1 .
\end{abstract}

\section{Introduction}

Settling velocity of particles has been used as a design parameter for different applications, such as particle separation, electrostatic precipitation, and spray drying. It is important to account for the wall effect on the terminal settling velocity of the particles in these applications. Wall effect has a retarding effect on the settling velocity of particles due to presence of wall. Wall effect is typically represented in terms of wall factor, $U_{t} / U_{t, \infty}$, where $U_{t}$ is the particle terminal settling velocity in a finite column and $U_{t, \infty}$ is the particle terminal settling velocity in an infinite column. Extensive studies have been reported in the literature regarding the wall effect on the settling of spherical particles. ${ }^{1-13}$ However, studies of the wall effect on the settling of non-spherical particles are relatively scarce. ${ }^{14}$ Among the nonspherical particles, cylindrical particles have become increasingly important in processes such as papermaking, synthetic cellulose and dry powder inhalation. ${ }^{15-19}$

The settling velocity of cylindrical particle that has a length to diameter ratio $(l / d)$ less than 2 is found to follow the predictions of the wall effect models developed for spherical particles with the use of the volume equivalent diameter, $d_{v}{ }^{20}$ This adjusted model becomes invalid when the cylindrical particles have larger $/ / d$ ratios. However, cylindrical particles having an $l / d>10$ are found to be affected by wall effect by a larger extent than cylindrical particles having an $l / d<10 .^{21}$ Typically, a cylindrical particle tends to orientate and equilibrate to a horizontal position when it is settling in stagnant fluid in the absence of wall. $^{15,22,23}$ When the wall effect becomes significant, the equilibrium orientation of the

\footnotetext{
${ }^{1}$ To whom correspondence should be addressed

Email address: wmlau@ntu.edu.sg
} 
cylindrical particle changes. The change in particle orientation leads to a different projected area and drag coefficient. Most wall effect studies on cylindrical particles involve the development of drag coefficient correlations either by relating to the drag coefficient correlations of spherical particles with volume equivalent diameter, $d_{v}$ and sphericity, $\psi^{24-29}$ or by developing new drag coefficient correlations for particle having a fixed shape and orientation. $^{15,30,31}$

There are a number of studies investigating the wall effect on cylindrical particle using a more empirical approach. ${ }^{21,32}$ Linear relationships are proposed between $U_{t} / U_{t, \infty}$ and $d_{v} / D$, where $D$ is the column diameter. The linearity constant is found to vary with Reynolds

numbers and $l / d$ ratios. $^{21,32}$ However, these linear equations are only valid in the viscous flow regime and for a small $d_{v} / D$ range of 0.05 to 0.5 for $l / d<10$ and 0.03 to 0.19 for $l / d>10$.

In this work, the wall effect on the settling of cylindrical particles is experimentally investigated in cylindrical columns in the inertial regime $\left(\operatorname{Re}_{t, \infty}>500\right)$. The wall effect is described in the context of changing particle orientation and retarding motion of the settling cylindrical particle. A semi-empirical correlation is established to predict the wall factor on cylindrical particles.

\section{Experimental Section}

Settling velocities of different shape particles are investigated in cylindrical columns of various diameters. The schematic diagram of the experimental setup is shown in Figure 1. Cylindrical particles of various materials and $l / d$ ratios are used in this study. The settling columns are made of acrylic and 1 meter in height. The dimensions of the particles and columns are listed in Tables 1 and 2, respectively.

Water is used as the settling medium. Particles are dropped at the centre of the column just under the liquid surface to create minimum disturbance of the liquid medium. The particle settling motion is recorded by a high-speed camera (Olympus, I-speed) at a frame rate of $500 \mathrm{~Hz}$. Video is captured at the bottom of the column to ensure that the particle has attained the terminal settling velocity. Experiments show that most particles attain the terminal settling velocity within $200 \mathrm{~mm}$ from the liquid surface. Due to the constant reorientation of the particle during settling, a fluctuation of the settling velocity can still be observed even after the particle has attained the terminal settling velocity. As a result, an averaged particle terminal settling velocity is determined based on the time needed for the center of gravity of the particle to travel from $600 \mathrm{~mm}$ to $800 \mathrm{~mm}$ from the liquid surface. Multiple measurements of the terminal settling velocity for each particle are taken to ensure the repeatability of results.

\section{Results and Discussion}

Settling of cylindrical particles in confined circular cylinder columns Figure 2 shows a typical relationship between the wall factor of cylindrical particles, $U_{t} / U_{t, \infty}$ and the cylindrical particle diameter to column diameter ratio, $d / D$. $d / D$ is used to represent the effect of wall 
such that the wall factor can be in analogy to spherical particle settling that at $d / D=1, U_{t} / U_{t, \infty}$ $=0$. As shown in the figure, the settling of cylindrical particles can be divided into several stages and the settling behaviors at each of the stage are discussed separately.

Point $\boldsymbol{a}$ - At this point, the cylindrical particle can be considered to be free-settling in an infinite column of stagnant liquid medium without any wall effect $\left(U_{t} / U_{t, \infty}=1\right)$. Since it is known that the cylindrical particle will achieve a stable horizontal settling orientation at this stage, ${ }^{15,22,23}$ the terminal settling velocity of cylindrical particle in an infinite stagnant liquid medium can be calculated theoretically by applying force balance on the particle:

$$
\rho_{p} \frac{\pi d^{2} l}{4} g=\rho_{l} \frac{\pi d^{2} l}{4} g+\frac{U_{t, \infty}^{2} C_{D, \text { horizontal cylinder }} \rho_{l} d l}{2}
$$

where the left hand side of Eq. (1) represents the gravitational force and the two terms on the right hand side of Eq. (1) represents the buoyancy force and drag force, respectively. Eq. (1) can be re-arranged to obtain an expression of the terminal settling velocity for cylindrical particle:

$$
U_{t, \infty}=\sqrt{\frac{\pi d g\left(\rho_{p}-\rho_{l}\right)}{2 C_{D, \text { horizontal cylinder }} \rho_{l}}}
$$

It can be seen that the terminal settling velocity for cylindrical particle is independent of the length of the particle. The drag coefficient is found to be 1.17 for $\operatorname{Re}>500{ }^{33}$ The $U_{t, \infty}$ calculated using Eq. (2) is also used as basis for the determination of the wall factor, $U_{t} / U_{t, \infty}$.

From point a to point $\boldsymbol{b}$ - When the length of cylindrical particle becomes appreciable compared to the diameter of the column, the presence of column wall causes a retarding effect on the particle. A particle settling with rotational, translational and lateral motion is observed. ${ }^{34}$ The settling behavior of the particle is illustrated in Figure 3. At the particular starting particle orientation, one side of the particle is likely to be closer to the column wall than the other. The side of the particle closer to the column wall will experience a stronger retarding effect from the wall. The difference in the settling velocities of the two sides then causes a translational motion along the center of gravity of the particle. As the particle orientation deviates from a horizontal position, a lateral motion pulls the particle away from the column wall. The lateral motion will cause the other side of particle to move closer to the wall and experience a stronger wall effect. Thus, the translational motion in the opposite direction occurs. This translation and lateral motion repeat itself throughout the settling process with occasional rotational motion along the horizontal plane. As the particle length to column diameter ratio increases, the difference in wall effect on the two sides of the particle becomes larger. The lateral motion becomes more significant and collision between the particle and the column can be observed. The particle-wall collision further reduces the settling velocity of the particle. Thus, $U_{t} / U_{t, \infty}$ reduces with an increase in $d / D$.

From point $b$ to point $\boldsymbol{c}$ - When the particle length to column diameter ratio reached a critical value (point b), further reduction in column diameter will cause the particle to settle in an angle that the particle is unable to return to the original position. The particle will instead circulate around the column wall in a spiral settling motion as illustrated in Figure 4 . When a particle is settling in this orientation, the drag force acting on the particle will be smaller 
because the projected area of the particle is reduced. As the column diameter continues to decrease, it is reasonable to expect the particle to orient in a more inclined manner and further reduces the drag force. Therefore, $U_{t} / U_{t, \infty}$ increases with an increase in $d / D$ until point c.

From point c to point $d$ - Beyond point c, the particle settling motion remains spiral but the retarding effect from the wall becomes significant. As $d / D$ increases, the spiral motion turns into an oscillatory motion along the center of gravity of the particle due to the limited spiral area. The constant collision between the two ends of the particle with the column wall significantly reduces the settling velocity of the particle. The oscillation frequency also increases as $d / D$ increases. Thus, the settling velocity of the particle is reduced.

Point $\boldsymbol{d}$ - At this point, the diameter of the cylindrical particle is the same as the column diameter. Theoretically, the particle is unable to settle in such a column. Therefore, $U_{t} / U_{t \infty}$ becomes 0 .

Model Development It is anticipated that the wall effect on the settling of cylindrical particles is governed by two factors; one is the re-orientation of the cylindrical particle during settling while the other is the retarding effect due to the presence of column wall. The reorientation of the cylindrical can be described by the angle of the major axis of the cylindrical particle to the horizontal plane, termed $\theta$. The force balance on the cylindrical particle settling at an angle $\theta$ can be described by the equation:

$$
\rho_{p} \frac{\pi d^{2} l}{4} g=\rho_{l} \frac{\pi d^{2} l}{4} g+\frac{U_{t}^{2} C_{D, \text { inclined cylinder }} \rho_{l}\left(d l \cos \theta+\frac{\pi d^{2}}{4} \sin \theta\right)}{2}
$$

where $d l \cos \theta+\frac{\pi d^{2}}{4} \sin \theta$ in the second term on the right hand side is the projected area of the particle in the direction of settling. Re-arranging Eq. (3), the terminal settling velocity of a cylindrical particle at an angle $\theta$ can then be expressed as:

$$
U_{t}=\sqrt{\frac{\pi d^{2} l\left(\rho_{p}-\rho_{l}\right) g}{2 C_{D, \text { inclined cylinder }} \rho_{l}\left(d l \cos \theta+\frac{\pi d^{2}}{4} \sin \theta\right)}}
$$

Combining Eq. (4) and Eq. (2), the wall effect caused by the re-orientation of the cylindrical particle can be expressed as:

$$
\frac{U_{t}}{U_{t, \infty}}=\sqrt{\frac{C_{D, \text { horizontal cylinder }} d l}{C_{D, \text { inclined cylinder }}\left(d l \cos \theta+\frac{\pi d^{2}}{4} \sin \theta\right)}}
$$

The retarding effect due to the presence of column wall has been widely studied in literature for spherical particles. For simplicity, the correlation of wall factor developed by Munroe for the settling of spherical particles in the inertial regime has been adopted: ${ }^{8}$ 


$$
\frac{U_{t}}{U_{t, \infty}}=1-\left(\frac{d}{D}\right)^{\frac{3}{2}}
$$

Thus, the overall wall factor on the settling of cylindrical particle can be expressed as:

$$
\frac{U_{t}}{U_{t, \infty}}=\sqrt{\frac{C_{D, \text { horizontal cylinder }} d l}{C_{D, \text { inclined cylinder }}\left(d l \cos \theta+\frac{\pi d^{2}}{4} \sin \theta\right.}\left[1-\left(\frac{d}{D}\right)^{\frac{3}{2}}\right]}
$$

The drag coefficient of horizontal cylinder at $\operatorname{Re}>500$ is $1.2 .^{33}$ The drag coefficient for the extreme position of an inclined cylinder would be the same as that of a disk, i.e. 1.17 at $\operatorname{Re}>$

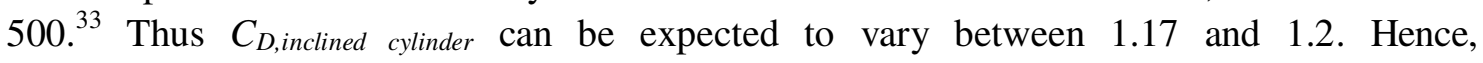
$\frac{C_{D, \text { horizontal cylinder }}}{C_{D \text {,inclined cylinder }}}$ can be taken approximately as $1 . \theta$ is anticipated to be dependent predominately by $l$ when wall effect starts to be effective and then dependent on $d$ when $d / D$ is approaching 1 . It is also reasonable to assume $\theta$ to change in a similar manner as described in Figure 5. When $D$ is large, the cylindrical particle will settle in a horizontal orientation and $\theta$ is equal to 0 . Wall effect becomes effective when $D$ is approaching $l$. Therefore, $l / D$ is believed to be a more realistic term to describe the orientation of the cylindrical particle. As $l / D$ increases, the cylindrical particle changes the orientation to minimize the wall effect and causes $\theta$ to increase. When $D$ is approaching $d$, the cylindrical particle will essentially be settling vertically and $\theta$ will remain constant as $\pi / 2$. It is proposed that $\theta$ will take the empirical form of:

$$
\theta=\frac{\pi}{2}\left\{\frac{k_{1}\left(\frac{l}{d}\right)}{k_{1}\left(\frac{l}{d}\right)+\exp \left[-k_{2} l^{k_{3}}\left(\frac{l}{D}\right)\right]}\right\}
$$

where $k_{1}, k_{2}$, and $k_{3}$ are fitting parameters and $k_{1}\left(\frac{l}{d}\right)<<1$. The requirement $k_{1}\left(\frac{l}{d}\right)<<1$ is needed for the exponential function to generate the " $\mathrm{S}$ " shape relationship between $\theta$ and $l / D$. Eqs. (7) and (8) are then combined and fitted to the experimental data obtained in the study by the least square error method. $k_{1}, k_{2}$, and $k_{3}$ are found to be $0.005,7.0$ and -0.03 , respectively. It is noted that the $\theta$ in Eq. (8) is by no means a true representation of the actual particle settling orientation. It is in fact a parameter that incorporates several factors including the particle orientation, the drag coefficient, and the retarding wall effect on the inclined cylindrical particle, which are a challenge to determine individually. Nevertheless, the semiempirical correlation developed in this study for the wall effect on the settling of cylindrical particle can be described as:

$$
\frac{U_{t}}{U_{t, \infty}}=\sqrt{\frac{d l}{d l \cos \theta+\frac{\pi d^{2}}{4} \sin \theta}\left[1-\left(\frac{d}{D}\right)^{\frac{3}{2}}\right]}
$$


and

$$
\theta=\frac{\pi}{2}\left\{\frac{0.005\left(\frac{l}{d}\right)}{0.005\left(\frac{l}{d}\right)+\exp \left[-7.0 l^{-0.03}\left(\frac{l}{D}\right)\right]}\right\}
$$

where $\theta$ has the unit of radian and $l, d$, and $D$ have the unit of mm.

A parity plot shown in Figure 6 indicates that the model developed is able to predict a majority of the experimental data within $\pm 25 \%$ of error. Some extreme cases are still observed. Particularly, an over-prediction of the wall effect when the $U_{t} / U_{t, \infty}$ predicted is large while an under-prediction of the wall effect when the $U_{t} / U_{t, \infty}$ predicted is small. Based on the experimental observation, when $d / D$ is small, the cylindrical particle occasionally slips along the column wall without any spiral motion. The retarding effect of the column dominates the settling behavior and causes the $U_{t}$ to be lower than the model prediction. The under-prediction of $U_{t} / U_{t, \infty}$ takes place normally when $l / d$ is large. Even though studies have indicated that cylindrical particle will achieve a stable horizontal settling orientation when there is no wall effect, ${ }^{15,22,23}$ the horizontal settling orientation is not stable for cylindrical particles having large $/ / d$. In this case, the cylindrical particle may settle at an inclined angle and achieve a $U_{t}$ higher than the model prediction. Nonetheless, Eqs. (9) and (10) are able to prediction the wall effect on the settling of cylindrical particles with reasonable accuracy.

Effect of particle length A comparison of the wall effect on the settling of cylindrical particles having a constant $d$ of $4 \mathrm{~mm}$ and various $l$ is shown in Figure 7 . It can be seen that as $l$ is increased, the retarding effect of the column diminishes and the particle deviates from the horizontal orientation at lower $d / D$. In addition, an increase in particle length increases the difference in the change in projected areas at the extreme settling orientations. Thus, the maximum $U_{t} / U_{t, \infty}$ also increases with $l$. When $d / D$ continues to increase beyond the maximum $U_{t} / U_{t, \infty}$, the particle is settling in almost a vertical orientation. An increase in $l$ will have minimal changes to the projected area. However, the gravitational force increases proportionally with an increase in $l$. Thus $U_{t} / U_{t, \infty}$ is higher for longer $l$.

Effect of particle diameter Similar to the wall effect on the settling of cylindrical particles having a constant $d$, Figure 8 shows that an increase in $U_{t} / U_{t, \infty}$ can be achieved by reducing $d$. A reduction in $d$ has more impact on the drag force than on the gravitational force and buoyancy force. As shown in Eq. (3), the drag force is proportional to the second order of $d$ at high $d / D$ ( $\theta$ close to 0$)$ and proportional to the first order of $d$ at low $d / D$ ( $\theta$ close to $\pi / 2)$. The gravitational force and the buoyancy force, on the other hand, are proportional to the second order of $d$ at all $d / D$. Therefore, a reduction in $d$ at low $d / D$ will have larger impact on $U_{t} / U_{t, \infty}$ than that at low $d / D$. In general, a change in $d$ with a constant $l$ or a change in $l$ with a constant $d$ can be viewed as a change in the aspect ratio, $l / d$ of the cylindrical particle. Further indicate in Figure 8, when $l / d$ is reduced to 1 , the model is reduced to the same wall effect model developed for spherical particles. It is in agreement with the literature that corrections developed for spherical particles can reasonably predict to the settling of cylindrical particles having an aspect ratio less than $2 .^{20,35,36}$ 
Effect of particle size with constant aspect ratio A comparison of the wall effect on the settling of cylindrical particles having a constant $l / d$ ratio of 4 but various diameters and lengths is shown in Figure 9. It can be seen that $U_{t} / U_{t, \infty}$ is only a function of $l / d$ and independent of the actual $d$ and $l$ when the particle settling orientation is essentially constant at low $d / D$ and high $d / D$. However, the actual $d$ and $l$ affect $U_{t} / U_{t, \infty}$ when the effect of wall on the particle is significant enough to change the orientation from horizontal to vertical. In the model development section, it has been explained that the particle orientation is governed by $l / D$ when $D>d$. Hence, for particles having the same $l / d$, an increase in $l$ will cause the particle orientation to happen at a higher $D$. However, the effective distance between the particle and the wall to have a noticeable wall effect should be relatively constant. Thus, the $l / D$ at which particle orientation happens, increases with an increase in $l$. After the particles become completely vertical, $l$ alone is not affecting the particle settling and $U_{t} / U_{t, \infty}$ becomes only a function of $l / d$ again.

\section{Concluding Remarks}

The wall effect on the settling velocities of cylindrical particles with various diameters and lengths are investigated in cylindrical columns. A semi-empirical model is developed by applying force balance and the incorporation of the particle orientation, the drag coefficient and the retarding wall effect on the settling particle. The model developed is found to give a good estimation of the wall effect on cylindrical particles having $l / d$ ratios of 4-21 and $\operatorname{Re}_{t, \infty}$ of 600-12100. Experimental results and the model predictions indicate that the presence of wall may change the particle orientation at low $d / D$ and allow the cylindrical particle to settling at a higher terminal velocity than that in the absence of wall. An increase in $l / d$ ratio generally allows the cylindrical particles to change its orientation at a lower $d / D$ and achieve a higher $U_{t} / U_{t, \infty}$. $U_{t} / U_{t, \infty}$ is a function of $l / d$ and independent of the individual $l$ and $d$ when the particle orientation is constant. Otherwise, an increase in $l$ or $d$ slightly delays the occurrence of particle re-orientation. It is expected that the developed model is beneficial to the development of more accurate design parameters for various applications when fibrous particles are involved.

\section{Acknowledgements}

Support by AcRF Tier1 Grant RG14/09 is gratefully acknowledged. 


\section{Nomenclature}

$C_{D}=$ drag coefficient

$d=$ cylindrical particle diameter or sphere diameter

$D=$ column diameter

$d_{v}=$ volume equivalent diameter

$g=$ gravitational constant

$l=$ cylindrical particle length

$\operatorname{Re}=$ particle Reynolds number

$\mathrm{Re}_{t, \infty}=$ particle Reynolds number at terminal settling velocity in an infinite column

$U_{t}=$ particle terminal settling velocity in a finite column

$U_{t, \infty}=$ particle terminal settling velocity in an infinite column

$\mu \quad=\quad$ fluid viscosity

$\rho_{l}=$ fluid density

$\rho_{p}=$ particle density

$\theta=$ parameter to combine the factors of particle orientation, drag coefficient and retarding wall effect

$\psi \quad=\quad$ particle sphericity 


\section{Literature Cited}

1. Bougas, A.; Stamatoudis, M., Wall factor for acceleration and terminal velocity of falling spheres at high Reynolds numbers. Chemical Engineering \& Technology 1993, 16, 314-317.

2. Brown, P. P.; Lawler, D. F., Sphere Drag and Settling Velocity Revisited. Journal of Environmental Engineering 2003, 129, (3), 222.

3. Di Felice, R., A relationship for the wall effect on the settling velocity of a sphere at any flow regime. International Journal of Multiphase Flow 1996, 22, (3), 527-533.

4. Di Felice, R.; Parodi, E., Wall effects on the sedimentation velocity of suspensions in viscous flow. AIChE Journal 1996, 42, (4), 927-931.

5. Fidleris, V.; Whitmore, R. L., Experimental determination of the wall effect for spheres falling axially in cylindrical vessels. British Journal of Applied Physics 1961, 12, 490-494.

6. Francis, A. W., Wall effect in falling ball method for viscosity. Physics 1933, 4, 403.

7. Kehlenbeck, R.; Di Felice, R., Empirical relationships for the terminal settling velocity of spheres in cylindrical columns. Chemical Engineering \& Technology 1999, 22, (4), 303-308.

8. Munroe, H. S., The english versus the continental system of jigging-is close sizing advantageous? Transactions of the American Institute of Mining and Metallurgical Engineers 1888, 17, 637-659.

9. Newton, I., Principia. Lib. II, Prop. XXXIX, Theor. XXXI. Cambridge University Press: Cambridge, 1687.

10. Clift, R.; Grace, J. R.; Weber, M. E., Bubbles, Drops, and Particles. Dover Publications: 1978.

11. Di Felice, R.; Gibilaro, L. G.; Foscolo, P. U., On the hindered settling velocity of spheres in the inertial flow regime. Chemical Engineering Science 1995, 50, (18), 3005-3006.

12. Chhabra, R. P.; Uhlherr, P. H. T.; Richardson, J. F., Some further observations on the hindered settling velocity of spheres in the inertial flow regime. Chemical Engineering Science 1996, 51, (19), 4531-4532.

13. Chhabra, R. P.; Agarwal, S.; Chaudhary, K., A note on wall effect on the terminal falling velocity of a sphere in quiescent Newtonian media in cylindrical tubes. Powder Technology 2003, 129, 53-58.

14. Chhabra, R. P.; Agarwal, L.; Sinha, N. K., Drag on non-spherical particles: an evaluation of available methods. Powder Technology 1999, 101, (3), 288-295.

15. Fan, L.; Mao, Z.-S.; Yang, C., Experiment on Settling of Slender Particles with Large Aspect Ratio and Correlation of the Drag Coefficient. Industrial \& Engineering Chemistry Research 2004, 43, (23), 7664-7670.

16. Hassan, M. S.; Lau, R., Effect of Particle Shape on Dry Powder Inhalation: Study of Flowability. AAPS Pharmscitech 2010, 10, (4), 1252-1262.

17. Hassan, M. S.; Lau, R., Flowability study of different particle shapes in a bifurcation system. Particuology 2010, 8, 51-59.

18. Esmen, N. A., Adhesion and aerodynamic resuspension of fibrous particles. Journal of Environmental Engineering 1996, 122, 379-383. 
19. Fults, K. A.; Miller, I. F.; Hickey, A. J., Effect of particle morphology on emitted dose of fatty acid-treated disodium cromoglycate powder aerosols. Pharmaceutical Development and Technology 1997, 2, 67-79.

20. Unnikrishnana, A.; Chhabra, R. P., An Experimental Study of Motion of Cylinders in Newtonian Fluids: Wall Effects and Drag Coefficient Canadian Journal of Chemical Engineering 1991, 69, 729.

21. Chhabra, R. P., Wall effects on terminal velocity of non-spherical particles in nonNewtonian polymer solutions. Powder Technology 1996, 88, (1), 39-44.

22. Hazzab, A.; Terfous, A.; Ghenaim, A., Measurement and modeling of the settling velocity of isometric particles. Powder Technology 2008, 184, (1), 105-113.

23. Becker, H. A., The effects of shape and Reynolds number on drag in the motion of freely oriented body in an infinite fluid. Canadian Journal of Chemical Engineering 1959, 37, 85.

24. Thompson, T. L.; Clark, N. N., A holistic approach to particle drag prediction. Powder Technology 1991, 67, 57-66.

25. Swamee, P. K.; Ojha, C. P., Drag Coefficient and Fall Velocity of Non-Spherical Particles Journal of Hydrologic Engineering 1991, 117, (4), 660-669.

26. Ganser, G. H., A rational approach to drag prediction of spherical and non-spherical particles. Powder Technology 1993, 77, 143-152.

27. Chien, S.-F., Settling velocity of irregularly shaped particles. SPE Drilling \& Completion 1994, 9, (4), 281-289.

28. Hartman, M.; Trnka, O.; Svoboda, K., Free settling of nonspherical particles. Industrial \& Engineering Chemistry Research 1994, 33, 1979-1983.

29. Heider, A.; Levenspiel, O., Drag coefficients and terminal velocity of spherical and nonspherical particles. Powder Technology 1989, 58, (1), 63-70.

30. Christiansen, E. B.; Barker, D. H., The effect of shape and density on the free settling of particles at high Reynolds numbers. AIChE Journal 1965, 11, (1), 145-151.

31. Rajitha, P.; Chhabra, R. P.; Sabiri, N. E.; Comiti, J., Drag on non-spherical particles in power law non-Newtonian media. International Journal of Mineral Processing 2006, 78, 110-121.

32. Chhabra, R. P., Wall effects on free-settling velocity of non-spherical particles in viscous media in cylindrical tubes. Powder Technology 1995, 85, (1), 83-90.

33. Fox, R. W.; Pritchard, P. J.; McDonald, A. T., Introduction to Fluid Mechanics. 7 ed.; Wiley: 2008.

34. Yin, C.; Rosendahl, L.; Knudsen Kaer, S.; Sorensen, H., Modelling the motion of cylindrical particles in a nonuniform flow. Chemical Engineering Science 2003, 58, (15), 3489-3498.

35. Pettyjohn, E. S.; Christiansen, E. B., Effect of particle shape on free-settling rates of isometric particles. Chemical Engineering Progress 1948, 44, (2).

36. Madhav, G. V.; Chhabra, R. P., Drag on non-spherical particles in viscous fluids. International Journal of Mineral Processing 1995, 43, (1-2), 15-29. 
Table 1. Dimensions of the different shape particles employed in the experiments

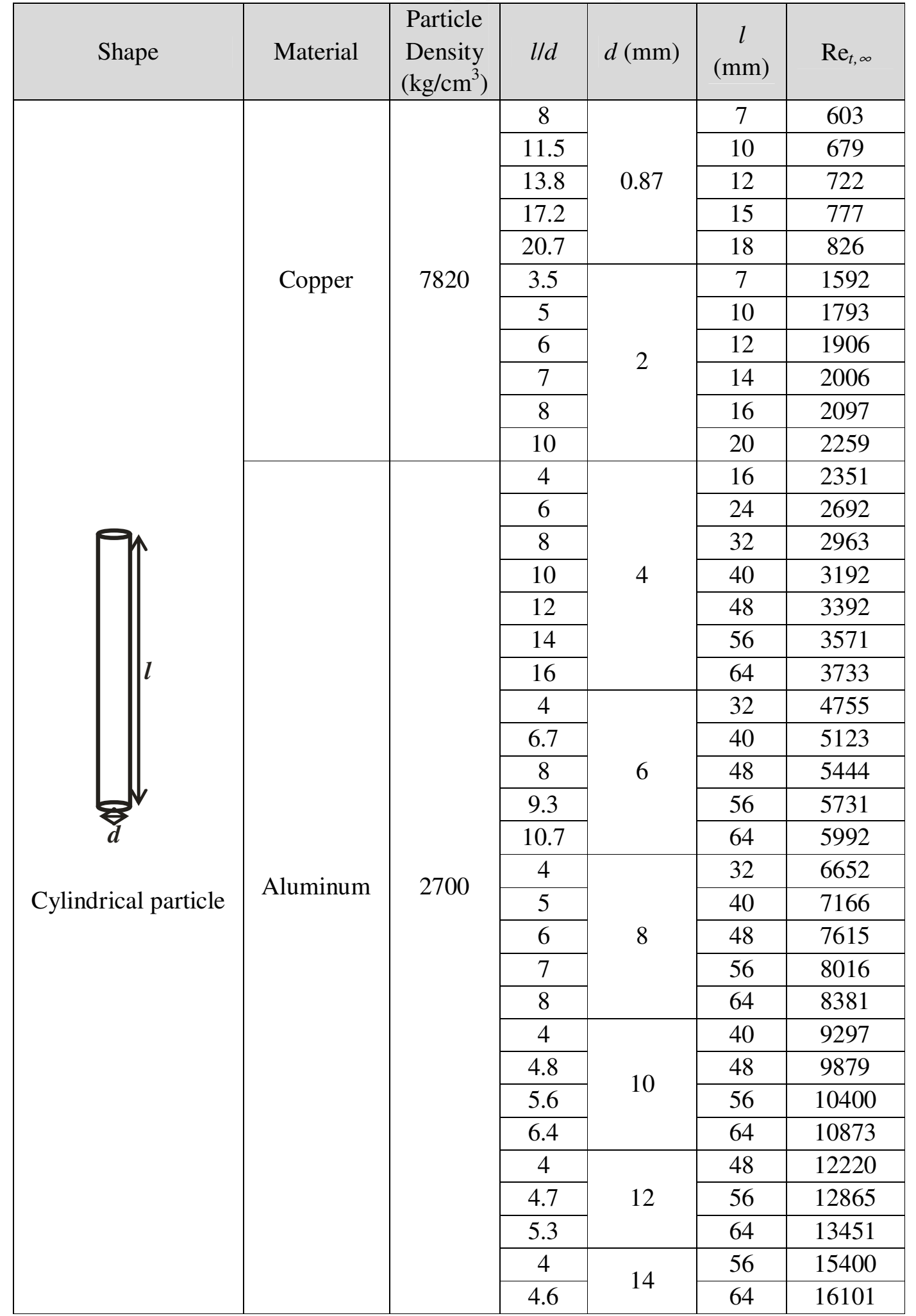


Table 2. Dimensions of the cylindrical columns used in this study

\begin{tabular}{|l|l|l|l|l|l|l|l|l|}
\hline Column No. & 1 & 2 & 3 & 4 & 5 & 6 & 7 & 8 \\
\hline $\begin{array}{l}\text { Inside } \\
\text { Diameter(mm) }\end{array}$ & 6 & 10 & 16 & 20 & 26 & 42 & 52 & 70 \\
\hline
\end{tabular}

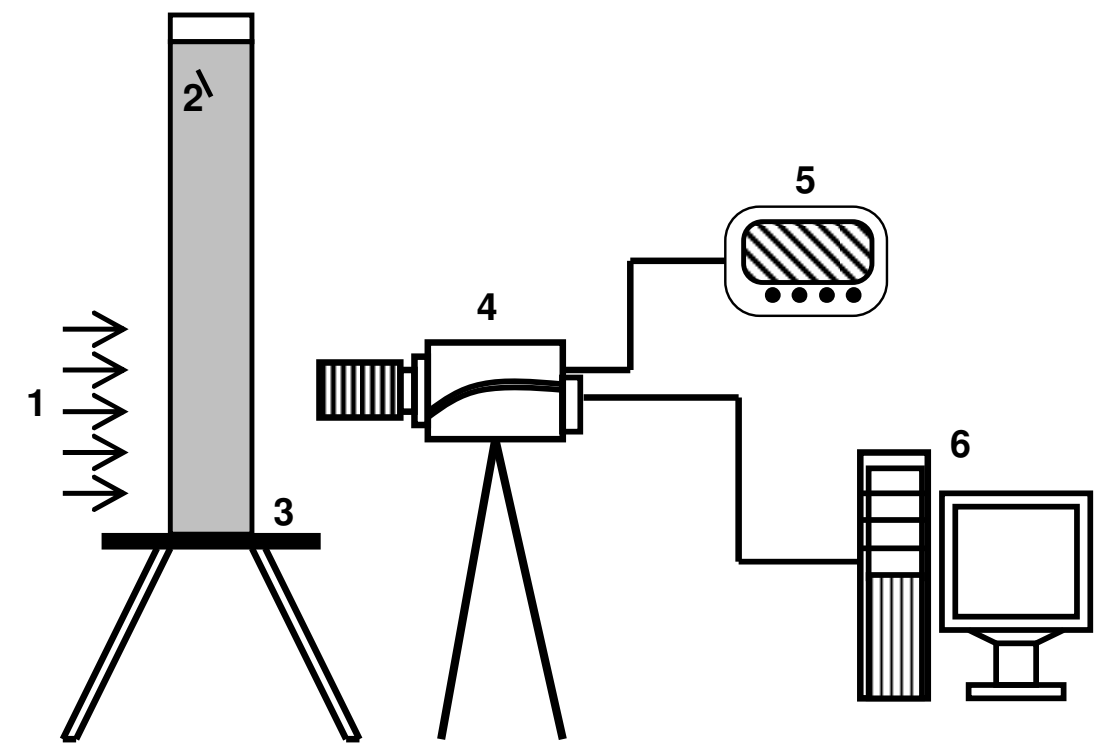

Figure 1. Schematic diagram of the experimental apparatus: 1) light source; 2) particle; (3) settling column; (4) high-speed camera; (5) monitor; (6) computer. 


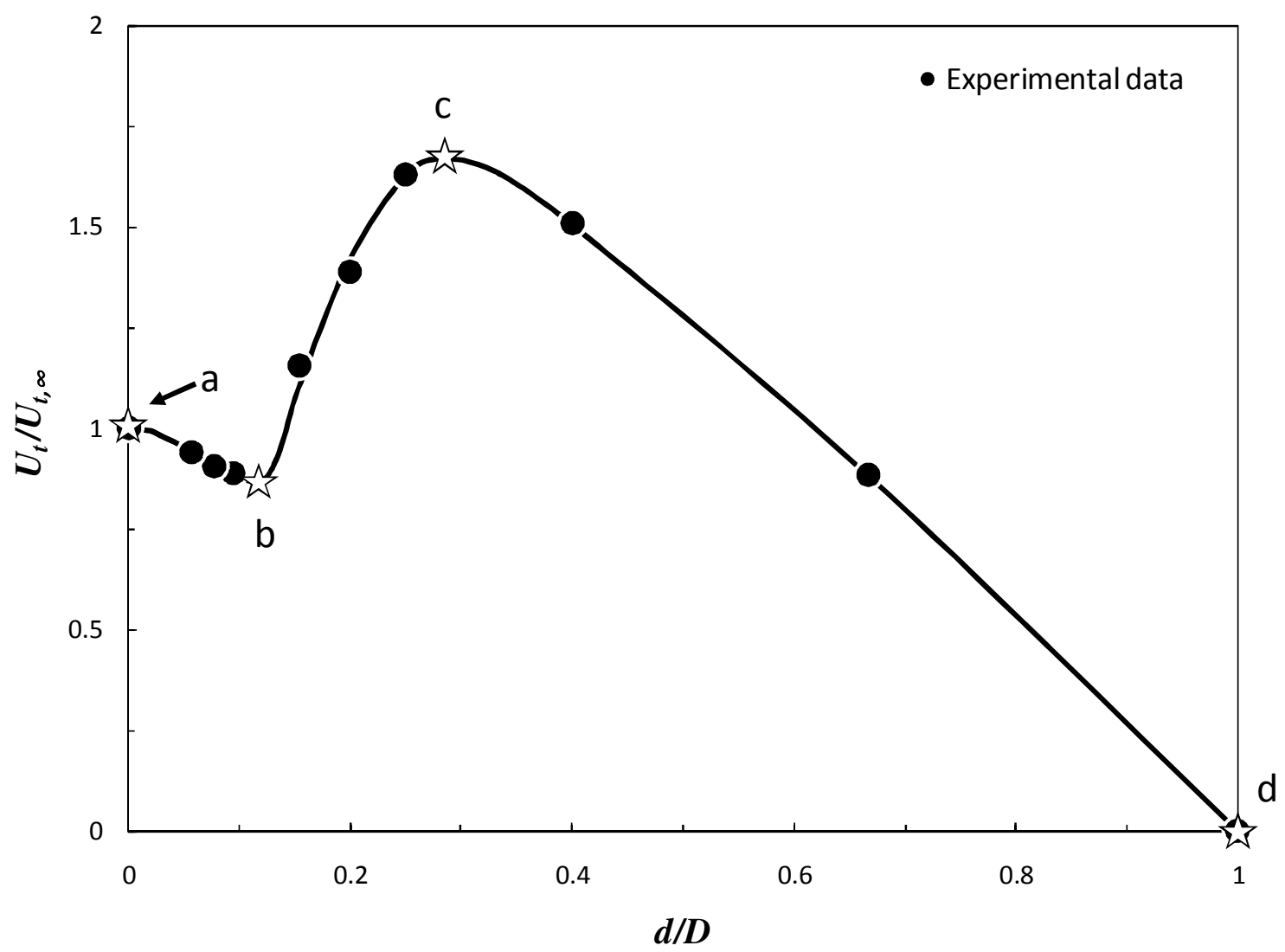

Figure 2. Typical wall effect on settling of cylindrical particles in a confined circular cylindrical column. 


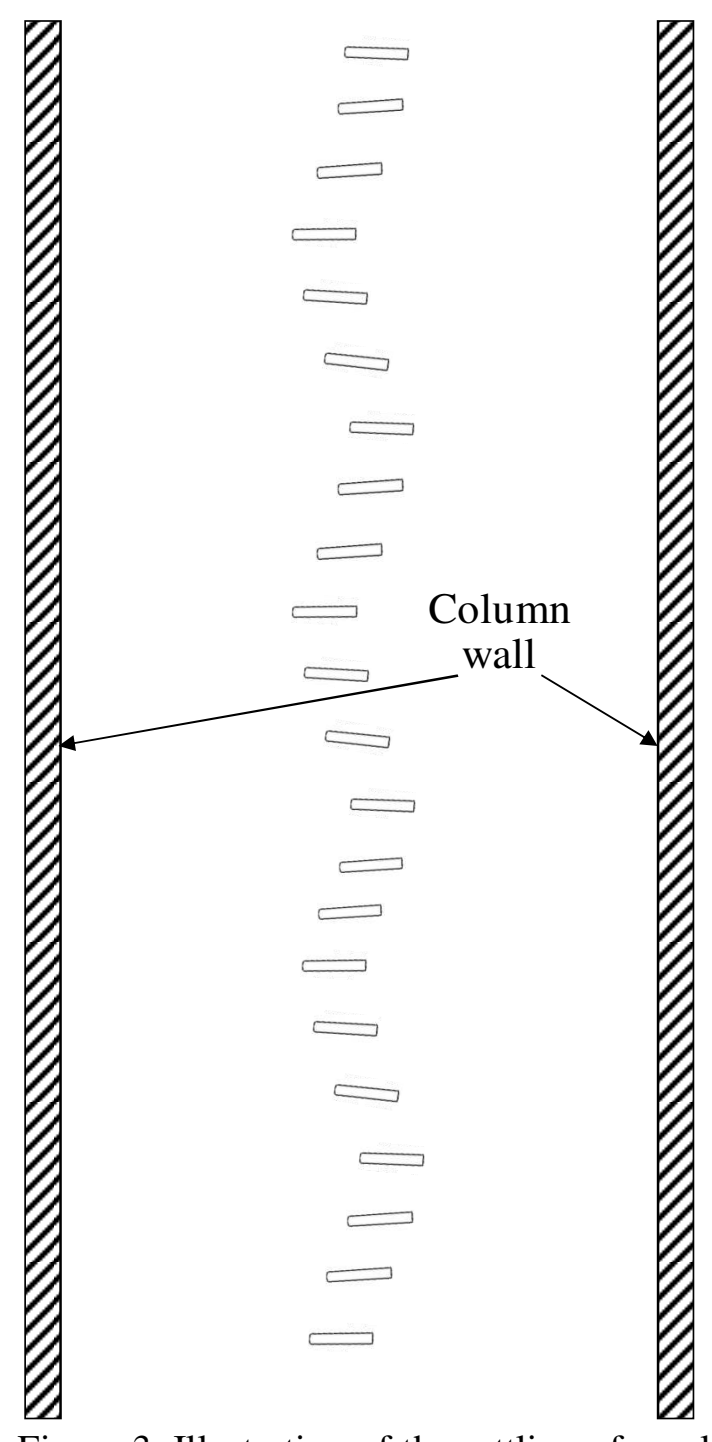

Figure 3. Illustration of the settling of a cylindrical particle in a confined cylindrical column with retarding wall effect.

14 


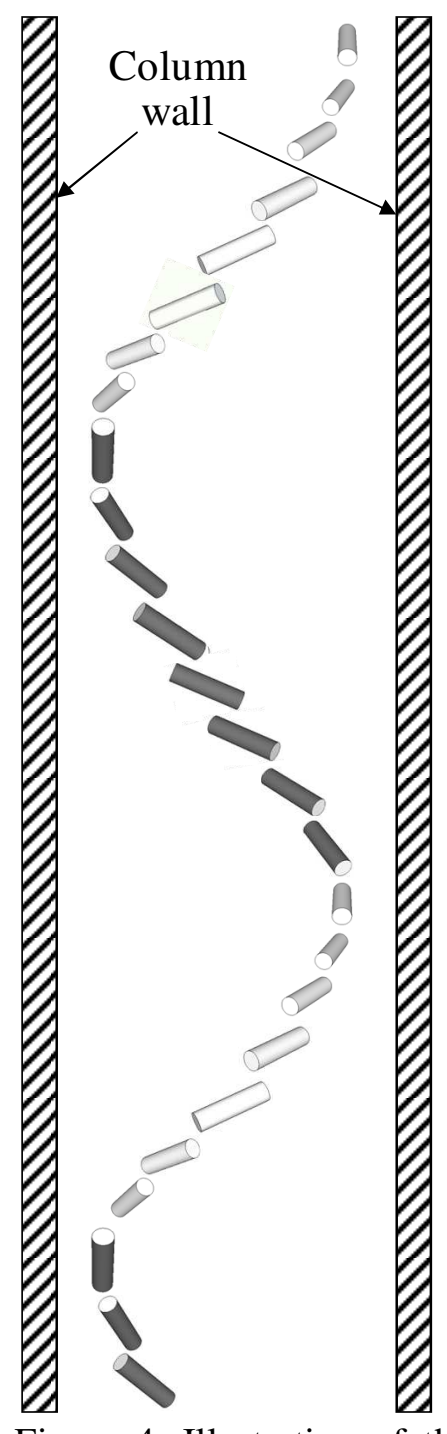

Figure 4. Illustration of the spiral settling motion of a cylindrical particle in a confined cylindrical column. 


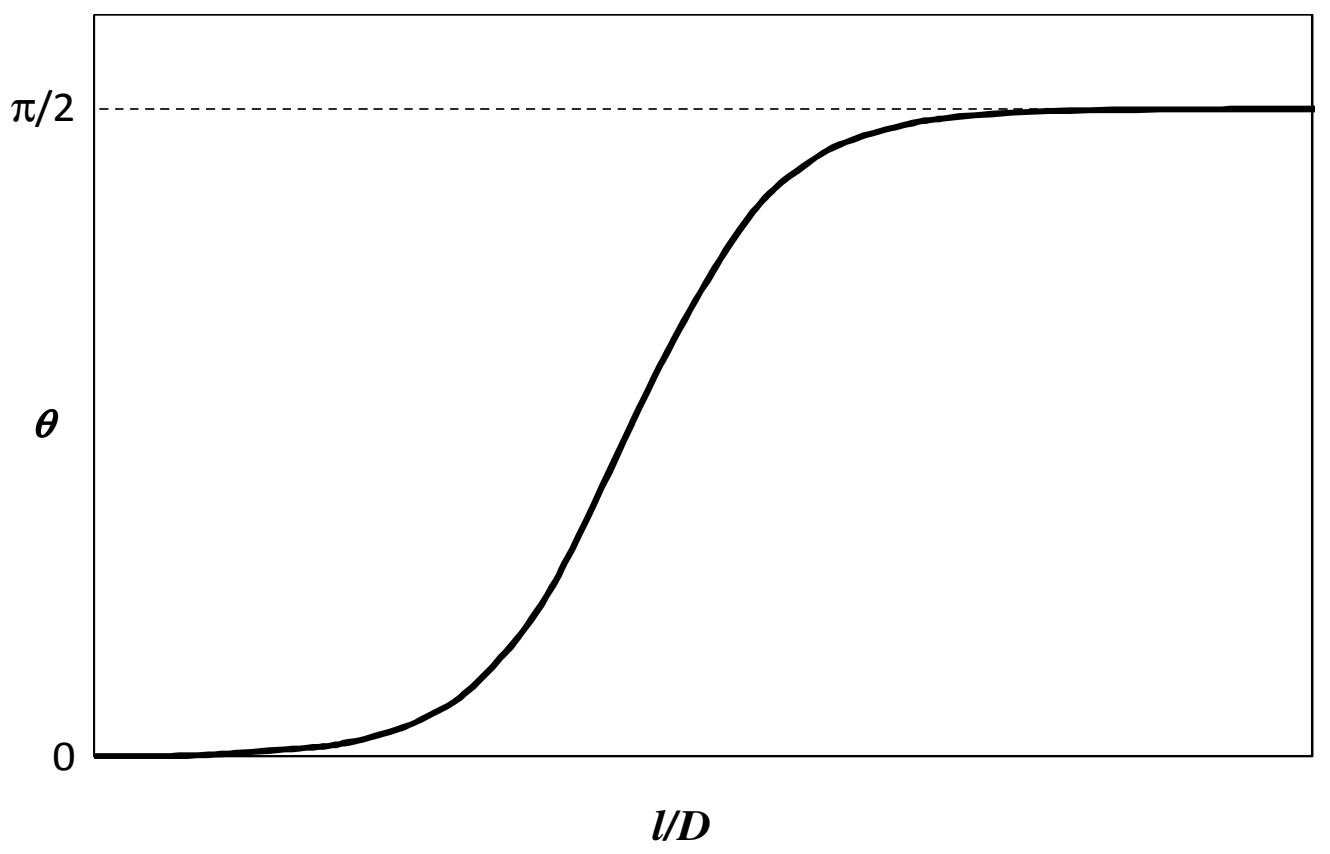

Figure 5. Illustration of the change in $\theta$ with $l / D$

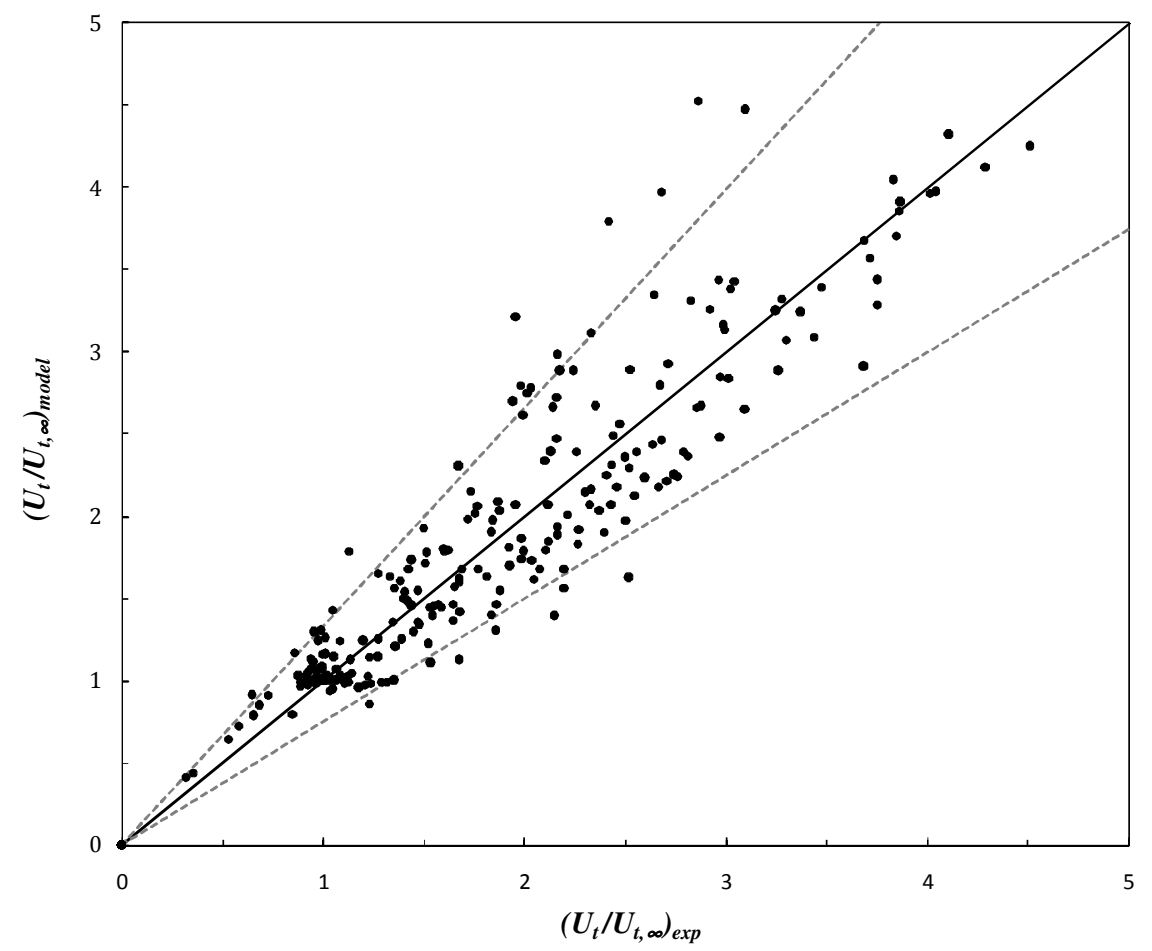

Figure 6. Comparison between the experimental data and model prediction of the wall effect on the settling of cylindrical particles. 


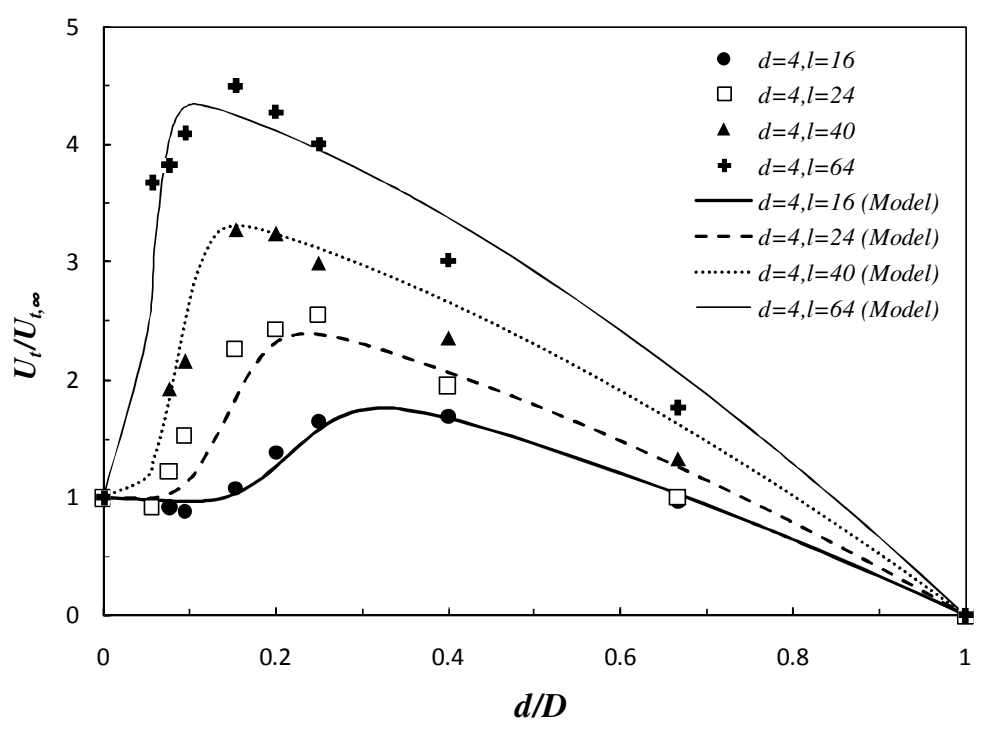

Figure 7. Comparison of the wall effect on the settling of cylindrical particles having a constant diameter and various lengths.

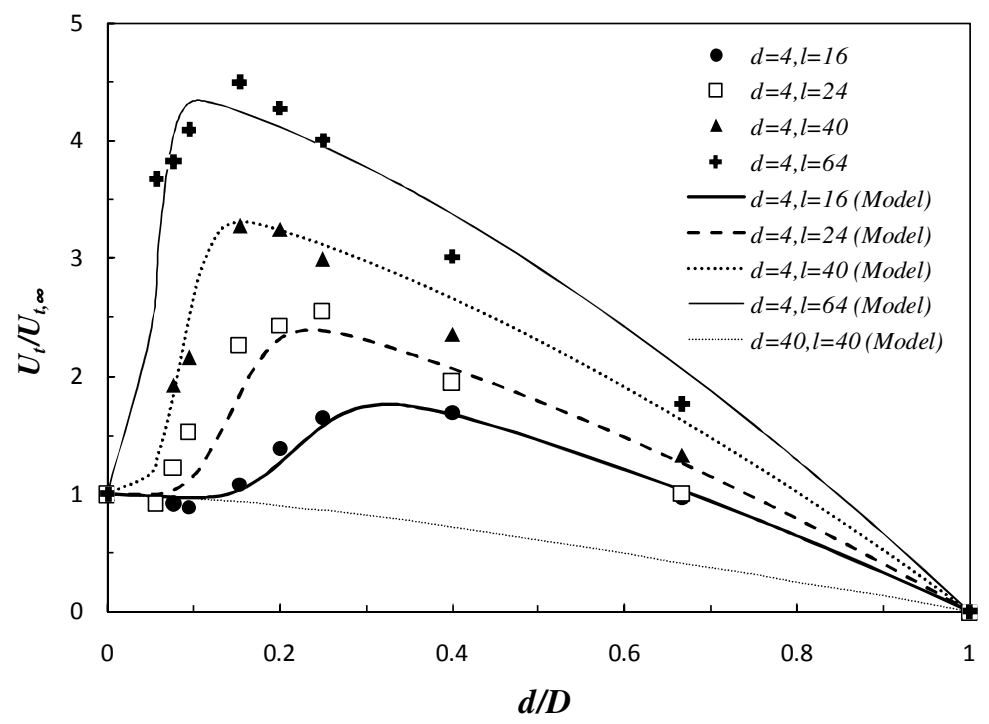

Figure 8. Comparison of the wall effect on the settling of cylindrical particles having a constant length and various diameters. 


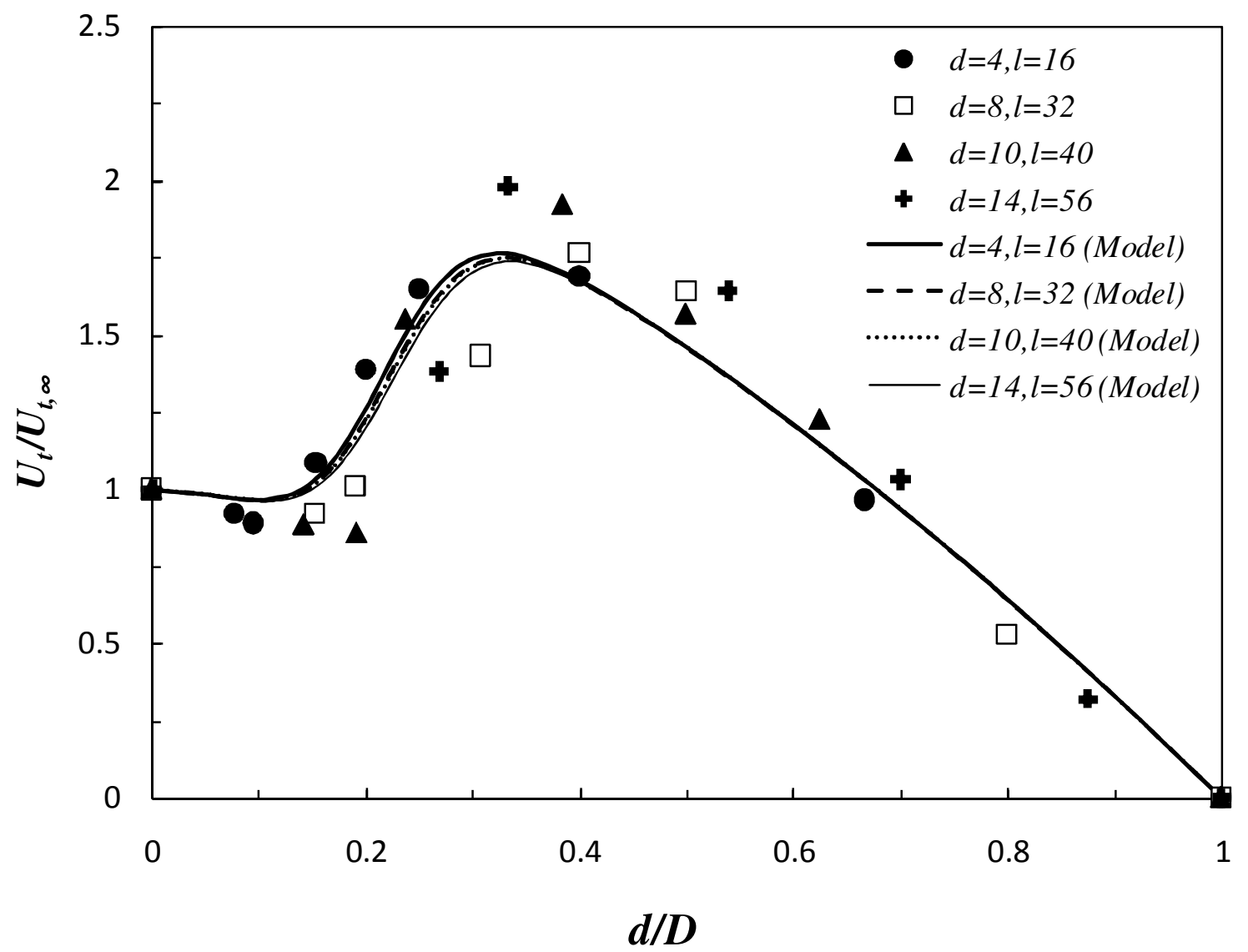

Figure 9. Comparison of the wall effect on the settling of cylindrical particles having a constant $l / d$ ratio but various diameters and lengths. 